OPEN ACCESS

Edited by:

Hai Xiong,

Shenzhen University, China

Reviewed by:

Yongxi Zhao,

Xi'an Jiaotong University, China

Weiwei Guo,

Nankai University, China

Chuan Zhang,

Shanghai Jiao Tong University, China

*Correspondence:

Feng $\mathrm{Li}$

fengli2018@tju.edu.cn

Specialty section

This article was submitted to

Biomaterials,

a section of the journa

Frontiers in Bioengineering and

Biotechnology

Received: 04 June 2021

Accepted: 25 June 2021

Published: 17 August 2021

Citation:

Lv Z, Zhu Y and Li F (2021) DNA

Functional Nanomaterials

for Controlled Delivery of Nucleic

Acid-Based Drugs.

Front. Bioeng. Biotechnol. 9:720291.

doi: 10.3389/fbioe.2021.720291

\section{DNA Functional Nanomaterials for Controlled Delivery of Nucleic Acid-Based Drugs}

\author{
Zhaoyue Lv, Yi Zhu and Feng Li*
}

Key Laboratory of Systems Bioengineering (MOE), Frontiers Science Center for Synthetic Biology, School of Chemical Engineering and Technology, Tianjin University, Tianjin, China

Nucleic acid-based drugs exhibited great potential in cancer therapeutics. However, the biological instability of nucleic acid-based drugs seriously hampered their clinical applications. Efficient in vivo delivery is the key to the clinical application of nucleic acid-based drugs. As a natural biological macromolecule, DNA has unique properties, such as excellent biocompatibility, molecular programmability, and precise assembly controllability. With the development of DNA nanotechnology, DNA nanomaterials have demonstrated significant advantages as delivery vectors of nucleic acid-based drugs by virtue of the inherent nucleic acid properties. In this study, the recent progress in the design of DNA-based nanomaterials for nucleic acid delivery is summarized. The DNA nanomaterials are categorized according to the components including pure DNA nanomaterials, DNA-inorganic hybrid nanomaterials, and DNA-organic hybrid nanomaterials. Representative applications of DNA nanomaterials in the controlled delivery of nucleic acid-based drugs are exemplified to show how DNA nanomaterials are rationally and exquisitely designed to address application issues in cancer therapy. At the end of this study, the challenges and future development of DNA nanomaterials are discussed.

Keywords: nucleic acid-based drugs, drug delivery, DNA assembly, DNA nanomaterials, DNA nanotechnology

\section{INTRODUCTION}

With the development of genomics and the elucidation of the genetic mechanism of cancer pathogenesis, gene therapy has been a promising therapeutic strategy for a variety of diseases, such as genetic disorders and cancers (Vaughan et al., 2020). Nucleic acid-based drugs mainly include plasmid DNA, antisense oligonucleotides (ASOs), small interfering RNA (siRNA), singleguided RNA (sgRNA), messenger RNA (mRNA), and immunostimulatory nucleic acids (Jiang and Thayumanavan, 2020; Vaughan et al., 2020). Intracellular nucleic acid delivery allows to reprogram cells at the gene level and provides an effective strategy to control protein generation, thus realizing precise regulation on cell functions (Vaughan et al., 2020). Compared with small molecule therapeutics, nucleic acid drugs were hardly taken up by cells due to their large molecular weight and hydrophilic nature. Moreover, nucleic acid drugs could be rapidly degraded by nucleases in the plasma with half-lives of several minutes, causing off-target and immune reactions (Jiang and Thayumanavan, 2020). Therefore, nanocarriers are needed to improve the stability of nucleic acid 
drugs in the plasma and further facilitate their cellular uptake. In recent years, significant progress has been made in developing nanocarriers for nucleic acid delivery. In particular, DNA-based nanomaterials are emerging as nucleic acid delivery vectors and have been extensively explored.

DNA, which is traditionally regarded as a genetic molecule, possesses excellent sequence programmability, precise molecular recognition, and abundant biological functions (Li F. et al., 2019; Dong et al., 2020). In 1953, Waston and Crick first reported the double-helix structure of DNA, leading to an extensive study on the structure of DNA (Li F. et al., 2019; Zhang et al., 2019). Conventional Watson-Crick base pairing refers to cytosine[C] and guanine[G] pairing, and adenine [A] and thymine [T] pairing (Zhang et al., 2019). In the early 1980s, Seeman initially proposed that DNA could function as building tiles for precisely constructing ordered nanostructure based on the Watson-Crick base pairing principle, opening a new frontier of DNA nanotechnology (Yuan et al., 2019; Dong et al., 2020). Owing to its intrinsic excellent biocompatibility, biodegradability, addressability, and low toxicity, DNA-based nanomaterials showed a unique advantage in disease diagnosis and cancer therapy (Wu et al., 2020). In particular, the excellent sequence programmability of DNA endowed the DNA nanomaterials with dynamic assembling behaviors, which facilitated spatiotemporally controllable assembly and release of nucleic acid drugs.

In this study, the recent progress on DNA-based nanomaterials for nucleic acid delivery is summarized. According to the components, the DNA nanomaterials are categorized including pure DNA nanomaterials, DNAinorganic hybrid nanomaterials, and DNA-organic hybrid nanomaterials (Figure 1). Representative applications of the DNA nanomaterials in the controlled delivery of nucleic acidbased drugs are exemplified to show how DNA nanomaterials are rationally and exquisitely designed to address nucleic acid drug delivery issues for cancer therapy. At the end of this study, the challenges and prospects of DNA nanomaterials for the delivery of nucleic acid-based drugs are fully discussed.

\section{PRUE DNA NANOMATERIALS}

Pure DNA nanomaterials are primarily constructed via selfassembly and DNA amplification technology (Zhang et al., 2019). Pure DNA nanomaterials take full advantage of selfassembly capability between DNA building blocks and nucleic acid drugs to realize controlled assembly and release of nucleic acid drugs as designed. For example, Lee et al. (2012) prepared DNA tetrahedral nanoparticles (NPs) via programmable selfassembly of short DNA fragments and therapeutic siRNAs (Figure 2A). Li et al. (2015) constructed a size-controllable and stimuli-responsive DNA nanohydrogel via self-assembly of three kinds of DNA building units for enhanced antisense DNA delivery. Long single-stranded DNA (ssDNA) produced by the rolling circle amplification (RCA) reaction was also used to construct DNA nanoribbons for intracellular gene silencing (Figure 2B; Chen et al., 2015). The long ssDNA

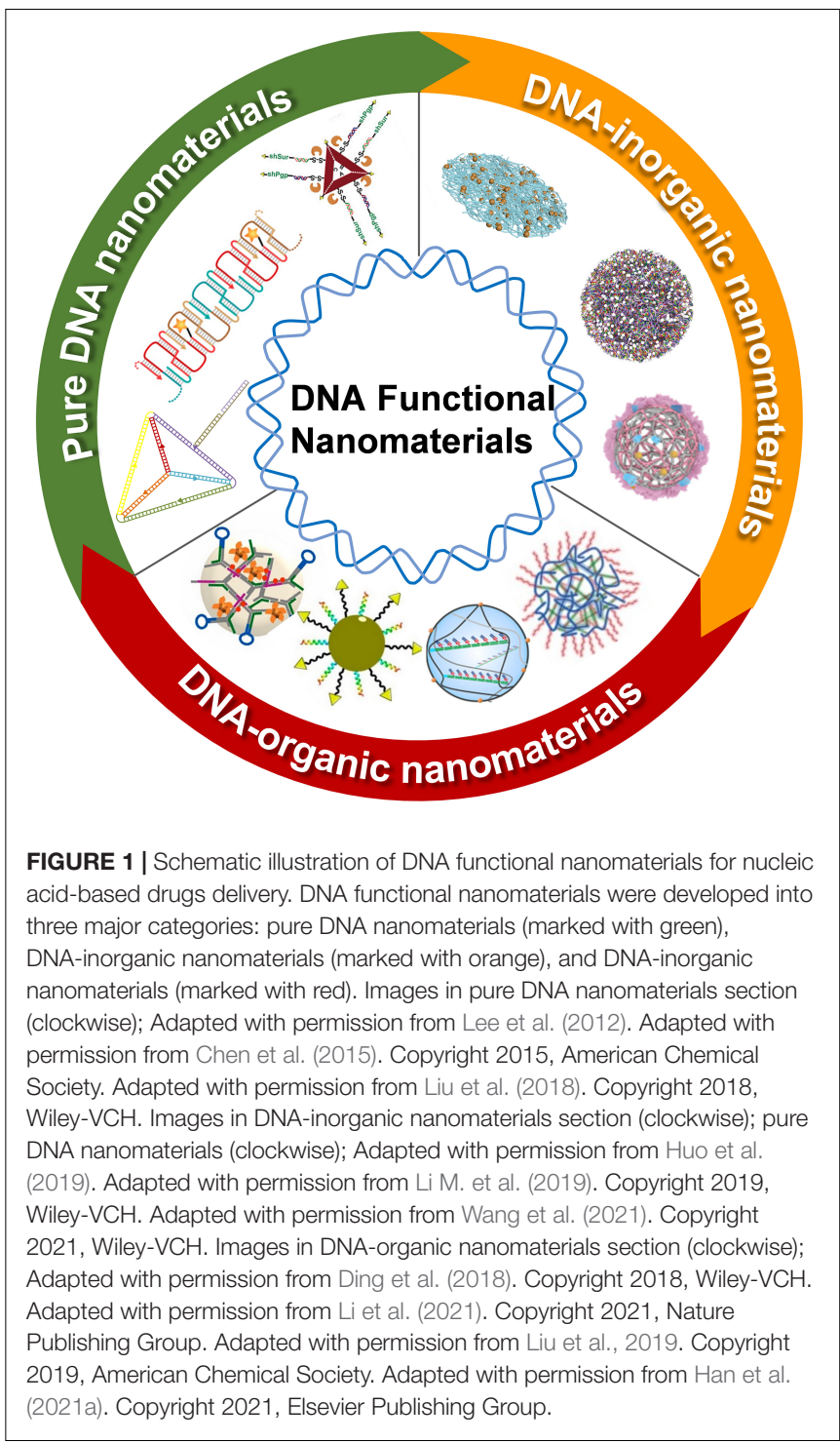

obtained by RCA reaction served as the backbone chain, and two types of DNA nanoribbons were obtained with the DNA origami method in which designer short ssDNA was used as staple chains. Due to its relatively high aspect ratio and rigid structure, DNA nanoribbons could effectively enter lung cancer cells (H460) via endocytosis and escape from lysosomes, thus achieving efficient siRNA delivery and gene silencing. This study combined DNA origami technology with RCA reaction and provided an ingenious method for designing a siRNA delivery system. Liu et al. (2018) constructed a tailored DNA origami for synergistic RNA interference (RNAi)-/chemotherapy of multidrug-resistant tumors (Figure 2C). Triangular DNA origami with cleavable disulfide capture strands and targeting aptamers was constructed in one pot via DNA assembly, followed by the doxorubicin loading. Subsequently, the linear small hairpin RNA transcription template targeted to the multidrug resistance-related genes (P-glycoprotein and survivin) could be tethered on the capture strand via base pairing. The tailored DNA 
A

Single-strand oligonucleotides

Wn

$\curvearrowright$

$\curvearrowright \curvearrowleft$

$\sim \sim \sim n$

iRNA with 3' overhang

$\sim \curvearrowleft$

$\underset{\text { One-step self-assembly }}{\stackrel{\longrightarrow}{\longrightarrow}}$

$\curvearrowright$

a

B

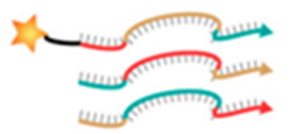

DNR-T
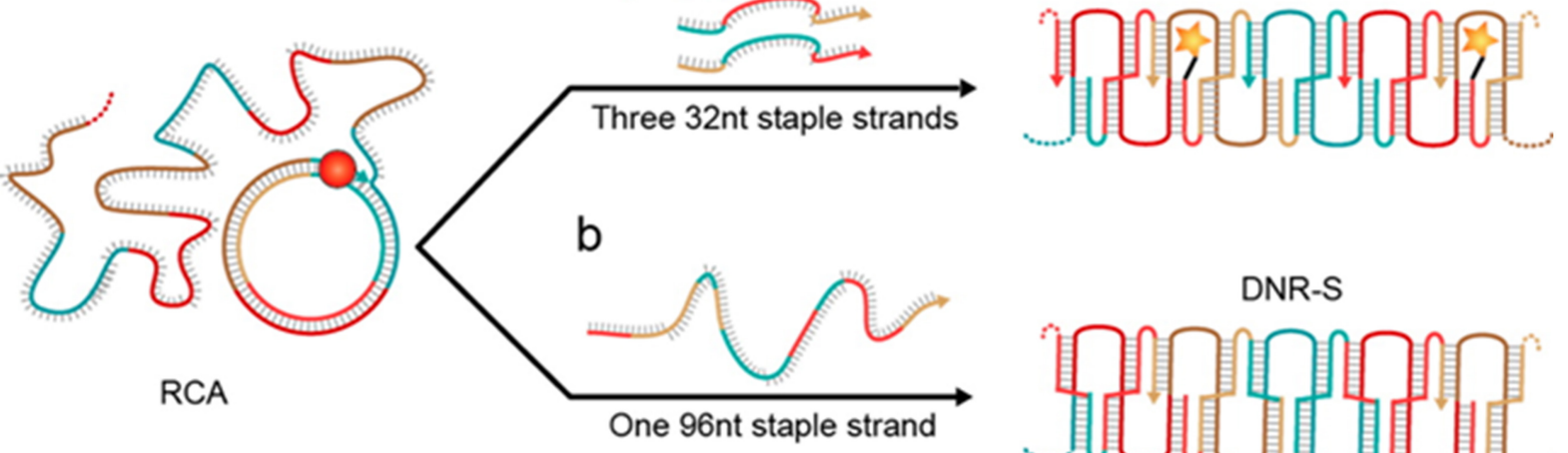

DNR-S

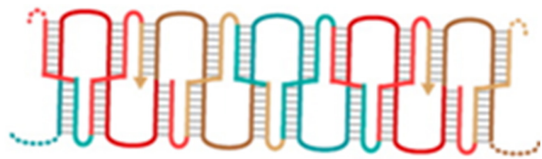

C

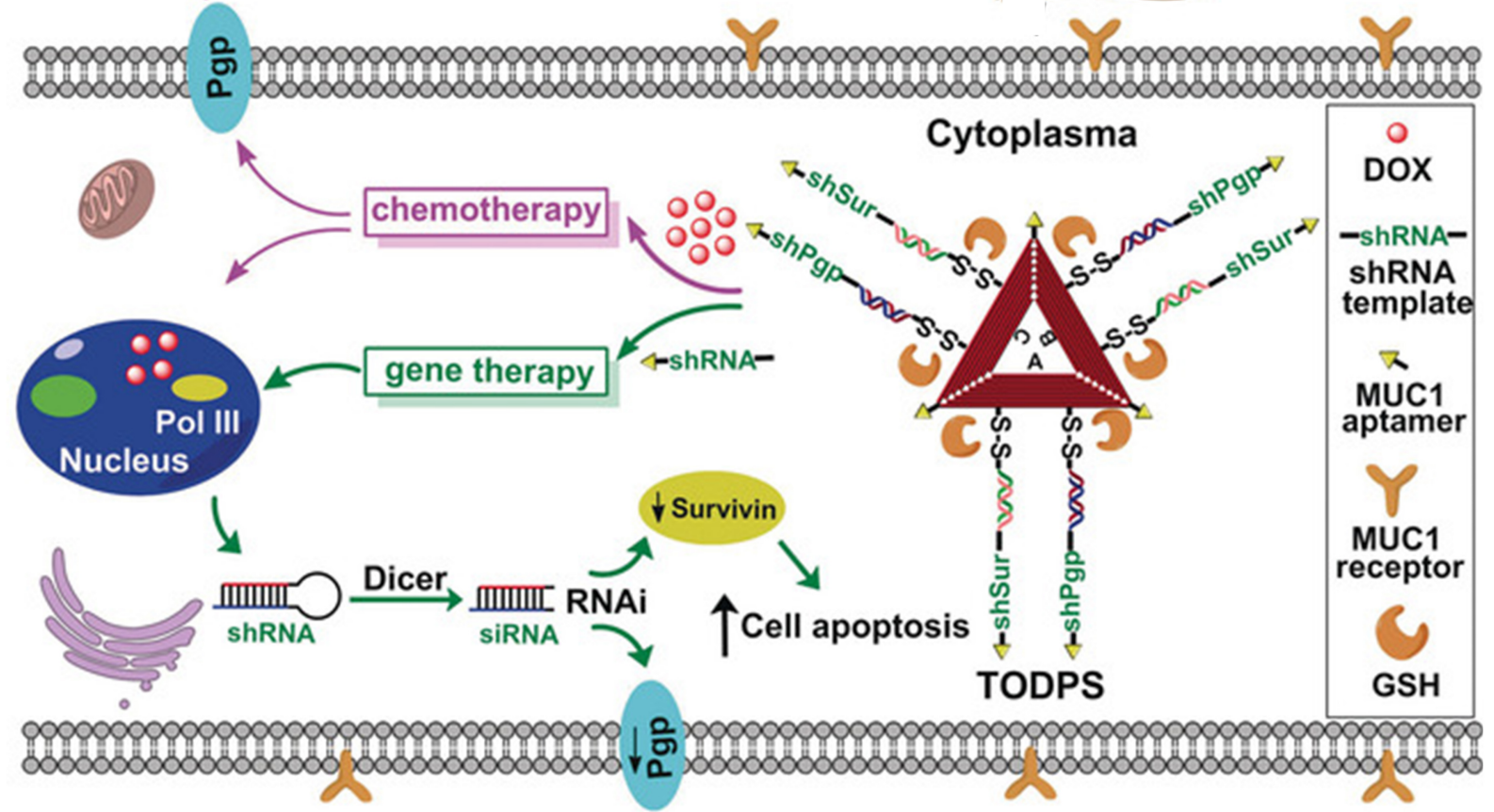

FIGURE 2 | Pure DNA nanoassembly for nucleic acid-based drugs delivery. (A) DNA tetrahedron for targeted in vivo siRNA delivery. Adapted with permission from ref. Lee et al. (2012). (B) DNA nanoribbons for siRNA delivery. Adapted with permission from ref. Chen et al. (2015). Copyright 2015, American Chemical Society. (C) DNA origami for synergistic RNAi-/chemotherapy. Adapted with permission from ref. Liu et al. (2018). Copyright 2018, Wiley-VCH. 
origami was internalized into breast cancer cells through mucin 1 (MUC1) targeting aptamer. With response to intracellular $\mathrm{pH}$ and glutathione (GSH), the DNA origami controllably released doxorubicin and gene drugs with a marked antitumor effect against multidrug-resistant tumors.

\section{DNA HYBRID NANOMATERIALS}

To improve stability and introduce multiple functional units that are not provided by pure DNA nanomaterials, hybrid DNA nanomaterials have been widely investigated for nucleic acid drug delivery (Hendrikse et al., 2019). Hybrid DNA nanomaterials combined the intrinsic recognition capability of DNA with extra functions of other materials to improve the performance in nucleic acid drug delivery. For instance, introducing inorganic nanocomponents into the DNA nanosystem could achieve desired optical and electronic features; polymer or supermolecule with targeting and stimuli-responsive properties has been integrated with DNA to obtain spatiotemporally controlled drug assembly and release ( $\mathrm{Li}$ F. et al., 2019). In this perspective, we summarized the developed hybrid DNA nanostructures including DNA-inorganic hybrids and DNAorganic hybrids for nucleic acid drug delivery. The challenges and opportunities of hybrid DNA nanomaterials in nucleic acid drug delivery were discussed.

\section{DNA-Inorganic Nanomaterials}

DNA could form hybrid nanomaterials with different moieties including inorganic NPs (Huo et al., 2019), mineral salt (Wang et al., 2021), and metal ions (Li M. et al., 2019). These DNAinorganic hybrids are usually conjugated or electrostatically adsorbed with nucleic acid drugs (Singh et al., 2010). The inorganic nanoparticles could protect nucleic acids and improve their stability in plasma (Jiang and Thayumanavan, 2020). Take gold $(\mathrm{Au}) \mathrm{NPs}$ as an example, ultrasmall ( $\sim \mathrm{nm})$ AuNPs with surface functionalization could deliver oligonucleotides to directly attack the cellular nucleus with no need for any targeting moiety (Huo et al., 2014). Inspired by this, Huo et al. (2019) constructed gold-DNA nanoflowers via DNAmediated self-assembly AuNPs for efficient gene silencing (Figure 3A). In their study, ultrasmall AuNPs were modified with cellular-myelocytomatosis viral oncogene $(c-m y c)$ silencing sequence (POY2T) which is a 23-nt (nucleotide) oligonucleotide via a ligand exchange method. Another designed ssDNA could complementarily hybridize with POY2T, thus blocking the $c$-myc oncogene silencing sequence via binding $c$-myc oncogene. Furthermore, POY2T modified AuNPs and the designed ssDNA would self-assemble into large-sized goldDNA nanoflowers, which would dissociate and release ultrasmall AuNPs upon near IR (NIR) irradiation. This study fully utilized both the high nuclear internalization efficiency of ultrasmall AuNPs and enhanced tumor accumulation/retention of goldDNA nanoflowers.

Metal-ligand coordination chemistry has emerged as another convenient strategy to synthesize nanomaterials with welldefined size and shape (Leininger et al., 2000). The key constituents of nucleic acids and nucleobases could coordinate with metal ions to form assemblies through coordination interactions (Amo-Ochoa and Zamora, 2014). For example, Li M. et al. (2019) reported a general approach for ferrous $\left(\mathrm{Fe}^{2+}\right)$ ions directing the assembly of DNA hybrid nanospheres via the coordination interaction (Figure 3B). By adjusting the molar ratio of metal ions and DNA, Fe-DNA nanospheres with adjustable sizes and controllable functions were obtained driven by coordination interactions between $\mathrm{Fe}^{2+}$ ions and DNA due to the abundance of phosphate-binding sites and oxygen and nitrogen atoms on the nucleobases. Cytosinephosphate-guanosine $(\mathrm{CpG})$, which could produce cytokines to inhibit cancer cell proliferation, was used as a model functional nucleic acid in this system. However, the obtained NPs showed low stability in fetal bovine serum (FBS), limiting its biological applications. Then, the mineralized NPs with a zeolitic imidazolate framework-8 (ZIF-8) exoskeleton to enhance serum stability. Compared with naked CpG, Fe-CpG-ZIF-8 showed enhanced cellular uptake efficiency and higher bioactivities, demonstrating an efficient high DNA delivery.

Another typical kind of DNA-inorganic molecule hybrid is DNA nanoflower with a flower-like morphology produced with the RCA method. The DNA nanoflower could maintain the stability of DNA in the bloodstream and load additional metal ions to active the biological functions of DNA (Lv et al., 2020). As an efficient isothermal enzymatic reaction, RCA could produce long preprogrammed DNA chains stabilized with pyrophosphate. By virtue of these programmability and intrinsic biocompatibility of DNA motif, DNA nanoflowers showed great potential in nucleic acid drug delivery. For example, Liu group constructed a DNA aptamer and hypoxia-inducible factor $1 \alpha(\mathrm{HIF}-1 \alpha)$ antisense DNA-integrated DNA nanoflower via RCA (Pan et al., 2020). The DNA nanoflower effectively inhibits HIF- $1 \alpha$ protein expression, demonstrating efficient HIF$1 \alpha$ antisense DNA delivery via DNA nanoflower. Wang et al. (2021) reported a bioinspired self-catabolic DNA nanosponge for efficient gene silencing via the rolling circle replication (RCR) (Figure 3C). The therapeutic DNAzyme (tDNAzyme) and self-motivated DNAzyme were designed as a template of RCR. tDNAzyme was masked by self-motivated responsive DNAzyme (rDNAzyme) during the RCR process. The obtained RCR scaffolds were modified with multivalent DNA aptamers (MUC1 aptamer), which could specifically target tumor cells. Besides, zinc oxide $(\mathrm{ZnO}) \mathrm{NPs}$ (acting as DNAzyme cofactor precursors) and proapoptotic cytochrome c protein (acting as chemotherapy agents) were encapsulated in the DNAzyme nanosponge. This multifunctional nanosponge was internalized into targeted tumor cells by virtue of MUC1 aptamer strand, in lysosome the encapsulated $\mathrm{ZnO}$ NPs were translated into zinc ion $\left(\mathrm{Zn}^{2+}\right)$, and the released $\mathrm{Zn}^{2+}$ could trigger the disassembly of rDNAzyme, leading to burst release of both rDNAzyme and cytochrome $\mathrm{c}$ for combined gene silencing and chemotherapy. This study fully combined DNA-inherent programmability and ZnO NP-promoting DNAzyme catalytic efficiency for enhanced DNAzyme-based therapy.

\section{DNA-Organic Nanomaterials}

Biomolecules, such as peptides (Chin et al., 2018), proteins (Pelegri-O'Day and Maynard, 2016), and nucleic acids 
A

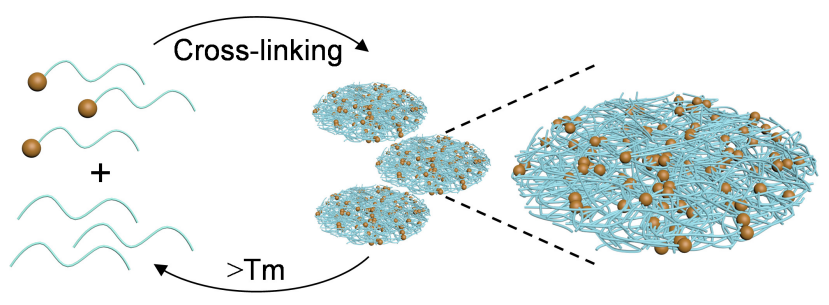

B

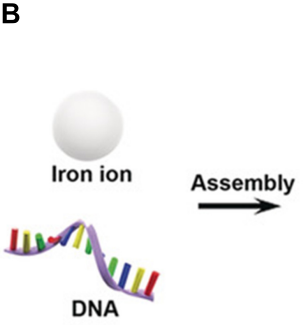

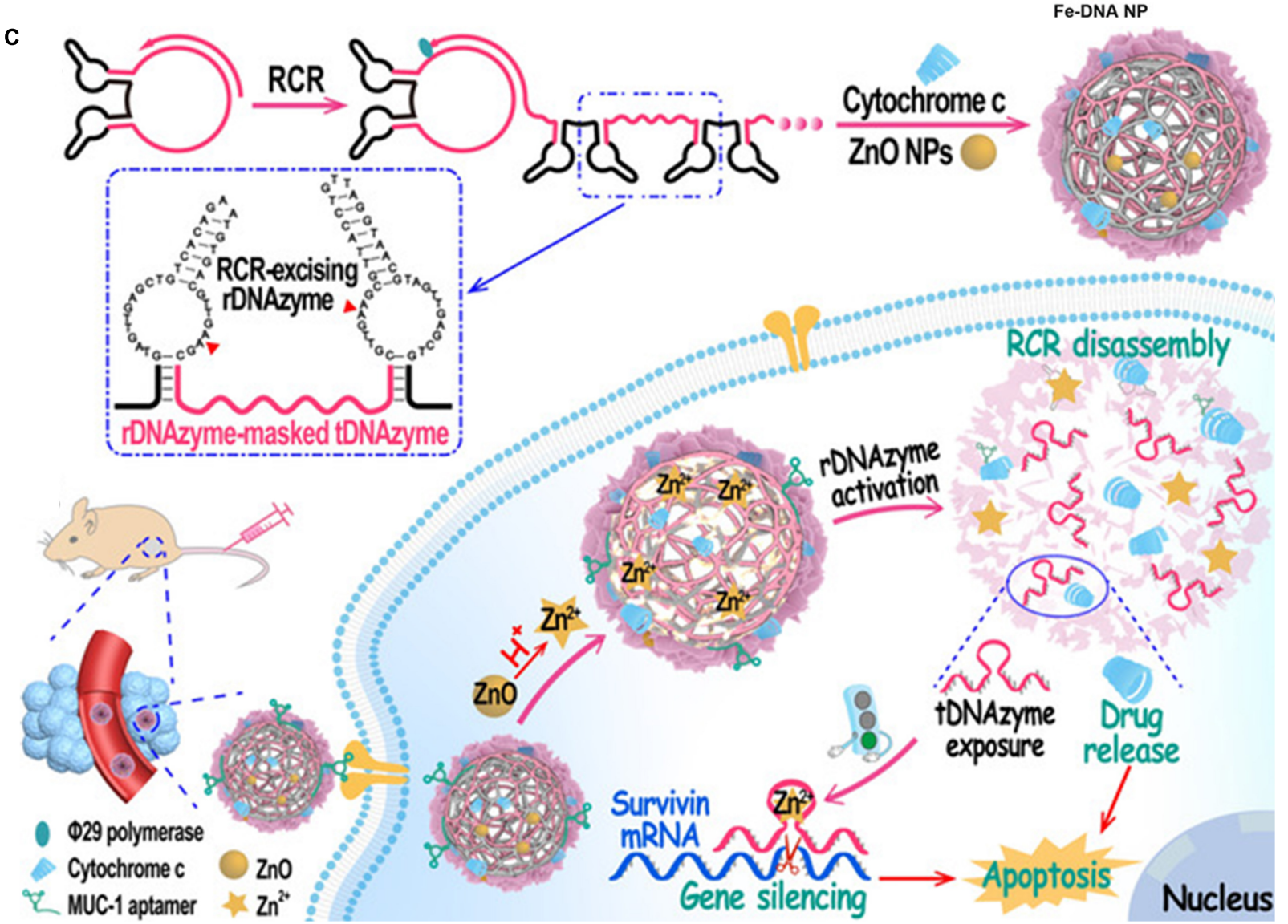

FIGURE 3 | DNA-inorganic nanoassembly for nucleic acid-based drugs delivery. (A) Gold-DNA nanoassembly for efficient gene silencing with controllable transformation. Adapted with permission from ref. Huo et al. (2019). Copyright 2019, Science Publishing Group. (B) Engineering Multifunctional DNA hybrid nanospheres through coordination-driven self-assembly for CpG delivery. Adapted with permission from ref. Li M. et al. (2019). Copyright 2019, Wiley-VCH. (C) DNA nanoflower for multifunctional DNAzyme delivery. Adapted with permission from ref. Wang et al. (2021). Copyright 2021, Wiley-VCH.

(Pan et al., 2017) could be integrated with synthetic polymers to form DNA-polymer hybrid nanomaterials with desirable functions (Luckerath et al., 2020). Compared with peptides and proteins, nucleic acids, such as precise biopolymers have unparalleled sequence programmability; therefore, the combination of nucleic acids with synthetic polymers could generate a variety of hybrid materials with extensive applications, especially in nucleic acid delivery (Luckerath et al., 2020).

In previous studies, siRNAs delivered by DNA tetrahedron were commonly exposed outside the nanostructures, which would inevitably make it degraded by ribonuclease (RNase). Conjugating nucleic acids with synthetic polymers could protect them from enzymatic degradation and prolong blood circulation. Ding et al. (2018) fabricated a cross-linked nucleic acid nanogel by using functional siRNAs as cross-linkers to direct selfassembly of DNA-grafted polycaprolactone (DNA-g-PCL) for effective siRNA delivery (Figure 4A). In their system, the siRNA was decorated with two single-stranded overhangs, which were complementary to the grated DNA in DNA- $g$-PCL, resulting in embedding siRNAs in the nanogel to avoid enzymatic degradation. The nanogel exhibited great knockdown efficiency of serine/threonine-protein kinase 1 (PLK1) in tumor tissues due to the enhanced cellular uptake. Moreover, the nanogel showed prolonged blood circulation with a half-life of ca. $1.14 \mathrm{~h}$, which 
A
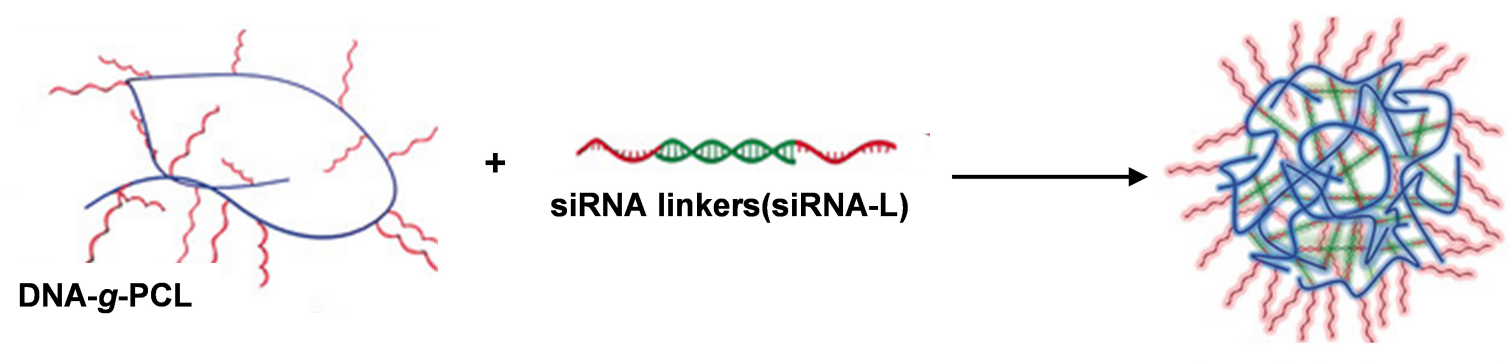

Crosslinked nanogel

B Formation of DNA cross-linked polymeric nanoframework (DPNF)

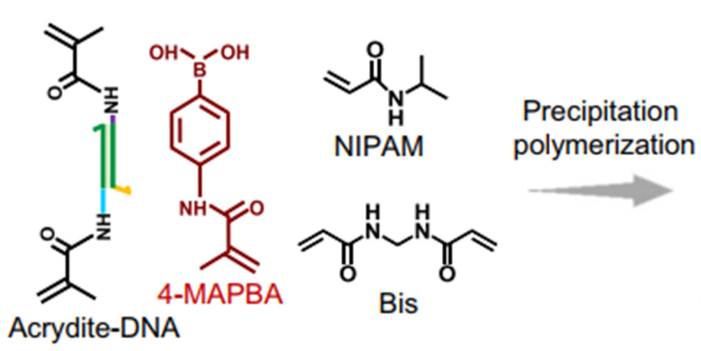
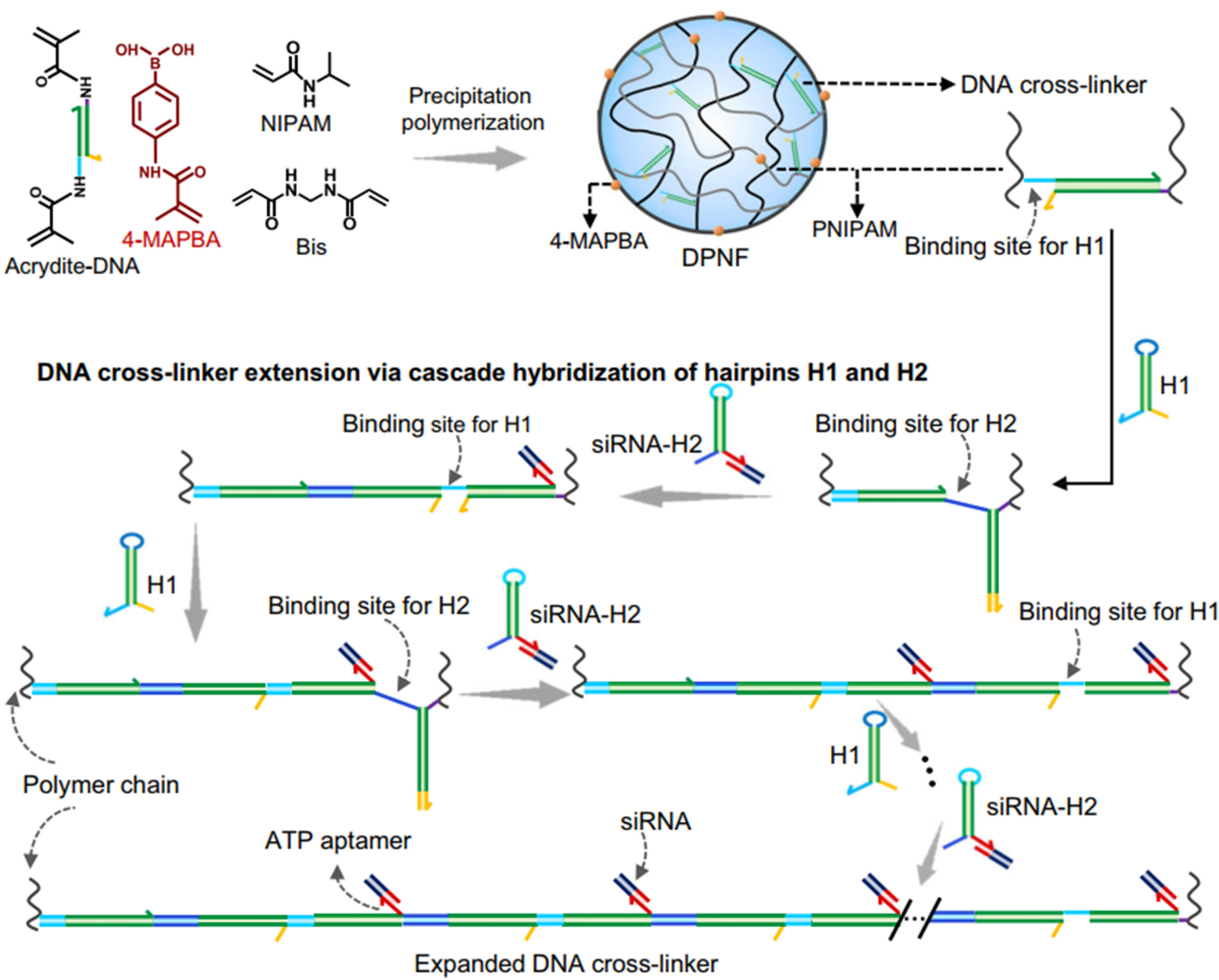

FIGURE 4 | DNA-polymer nanoassembly for efficient siRNA delivery. (A) Crosslinked nucleic acid nanogel for effective siRNA delivery. Adapted with permission from Ding et al. (2018). Copyright 2018, Wiley-VCH. (B) Cascade hybridization of hairpin DNA in polymeric nanoframework for precise siRNA delivery. Adapted with permission from ref (Li et al., 2021). Copyright 2021, Nature Publishing Group.

was much longer than those of naked siRNA (ca. 4 min) and Lipofectamine 2000 (Lipo2000) siRNA (ca. $10 \mathrm{~min}$ ).

We achieved controlling DNA assemble under polymeric nanoconfinement and develop a dynamic DNA assembly strategy in the confined space of polymer nanometers for precise siRNA delivery (Figure 4B; Li et al., 2021). Initially, we prepared DNA cross-linked polymeric nanoframeworks (DPNFs) on which tumor-targeting phenylboronates were decorated via precipitation polymerization. Cross-linked DNA served as the initiator that could drive cascade hybridization chain reaction (HCR) where hairpin monomers used potential energy to overcome the steric hindrance of DPNFs. All of the self-assembly processes were isothermal enzyme-free amplification. The sticky end of one DNA hairpin was designed elaborately as a triphosadenine (ATP) aptamer for efficient siRNA delivery in the DPNFs via base complementary pairing. 
A

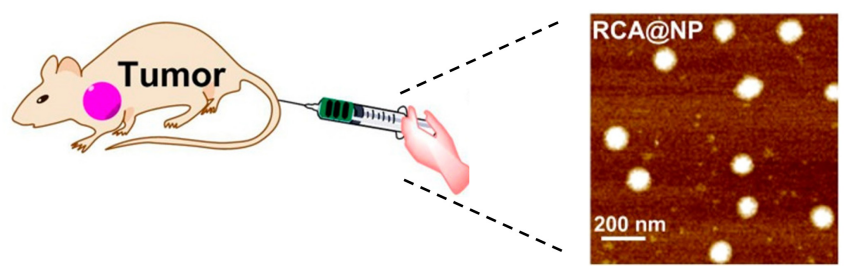

B

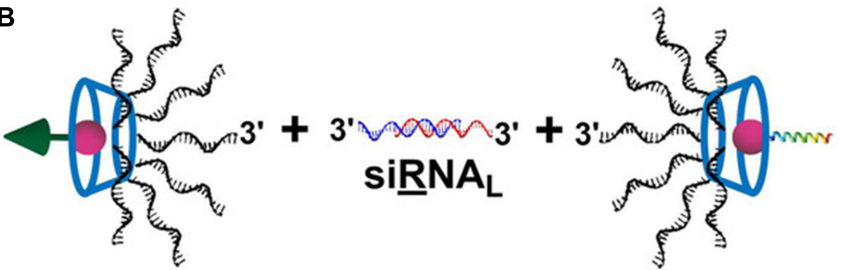

FA-7모1
HA-7소2

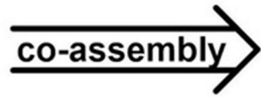

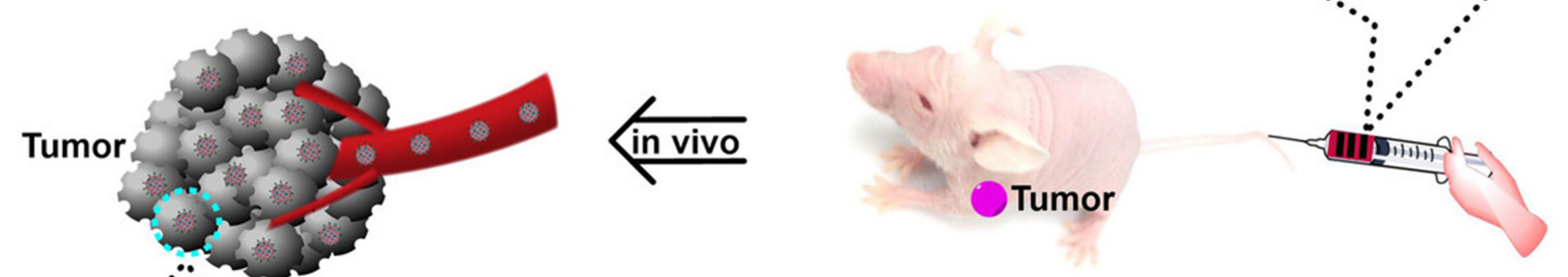
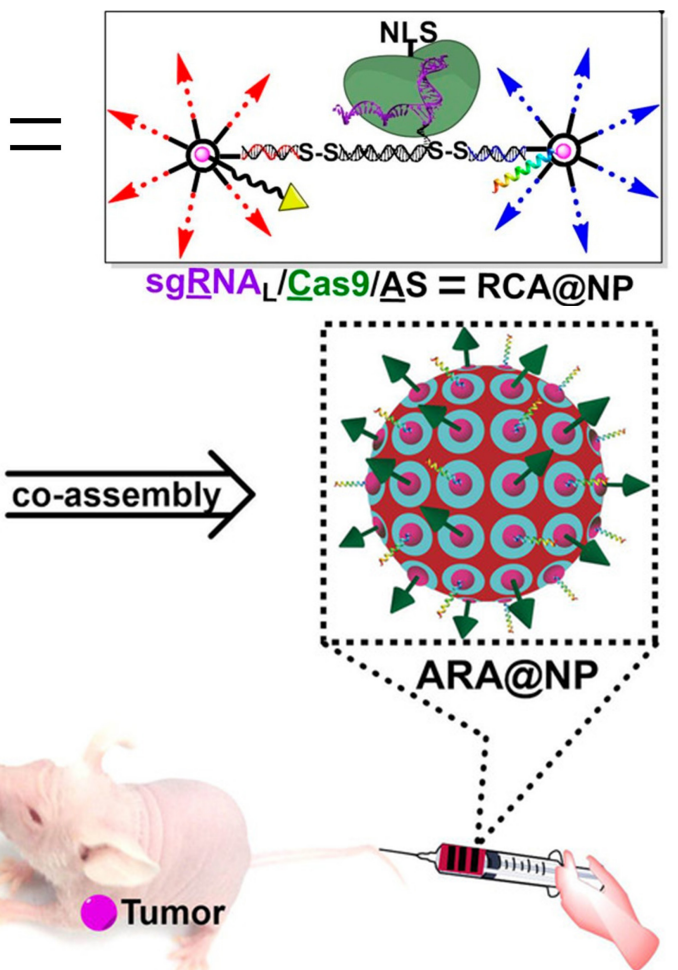

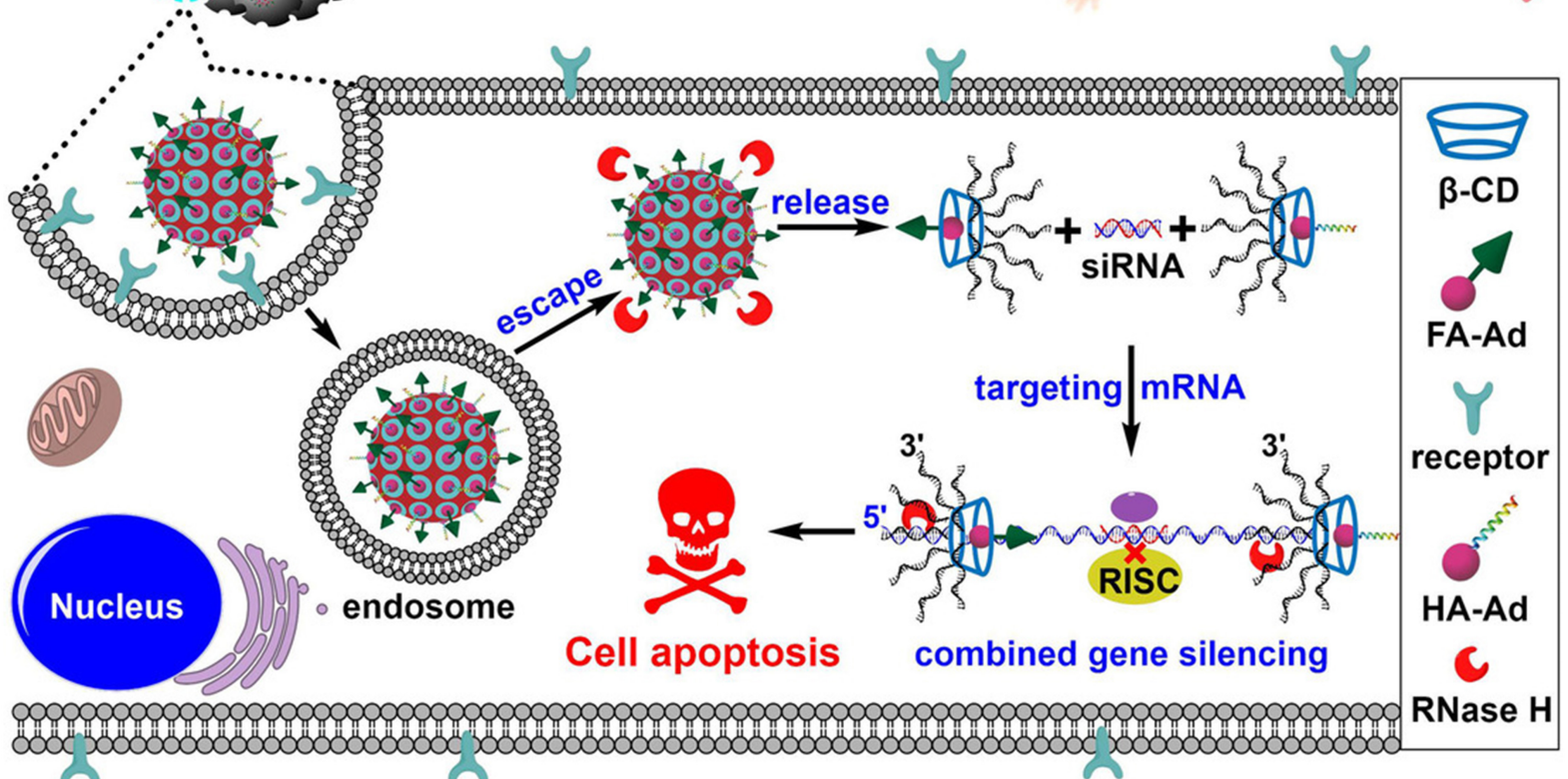

FIGURE 5 | DNA-supermolecule nanoassembly for nucleic acid delivery. (A) Multifunctional nucleic acid nanostructures for sgRNA/Cas9/antisense delivery. Adapted with permission from ref. Liu et al. (2019). Copyright 2019, American Chemical Society. (B) Crosslinked nucleic acid nanogel for effective siRNA delivery.

(B) Co-assembly of branched antisense and siRNA for combined gene silencing and tumor therapy. Adapted with permission from ref. Liu et al. (2021). Copyright 2020, Wiley-VCH.

Furthermore, in response to intracellular abundant ATP, siRNA was released especially in the cellular cytoplasm. Moreover, the functional group phenylboronate could endow tumor-targeting nanocarriers of DPNFs with actively recognizing the overexpressed sialic acid residues on the tumor cell membrane, thus improving the in vivo efficiency of gene delivery.

In contrast to chemical covalent bonds, supramolecular host-guest interactions provide another method for molecular recognition through non-covalent interactions with reversibility 
and stimuli-responsive nature ( $\mathrm{Hu}$ et al., 2018). $\beta$-cyclodextrin $(\beta-C D)$, the glucose heptamer, was a food additive approved by the Food and Drug Administration (FDA) (Ashton et al., 1996; Davis and Brewster, 2004). As one of the best supramolecular "host" molecules, $\beta$-CD could combine with adamantane (Ad), which is a typically "guest" molecule to form inclusion complexes due to the efficient molecular recognition and relatively strong binding interaction between $\beta$-CD and Ad (Davis and Brewster, 2004). Reji Varghese group reported a series of nano-to-micro-sized

A assembly reaction I gene loading

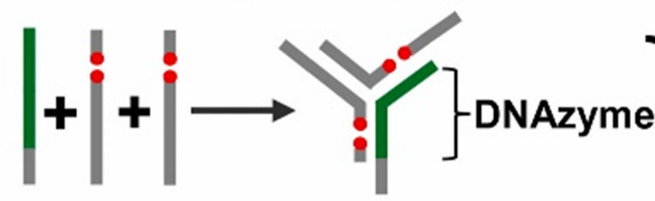

$\begin{array}{llll}Y_{1} & Y_{2} & Y_{3} & Y \text {-DNA }\end{array}$

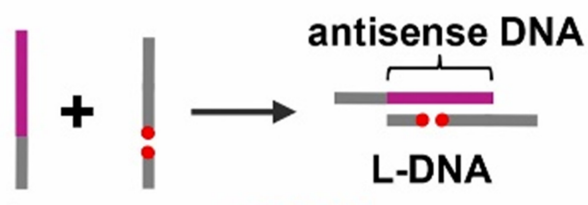

$\mathrm{L}_{1} \quad \mathrm{~L}_{2} \quad \bullet \mathrm{S}-\mathrm{S}$

TA
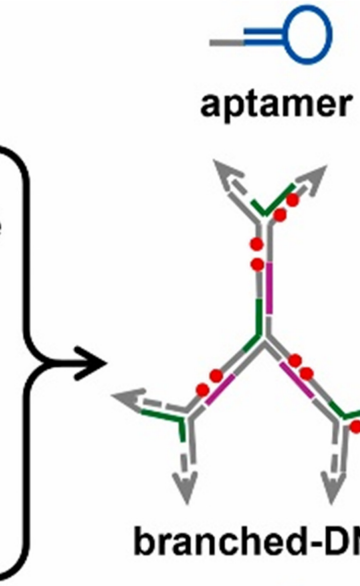

bran

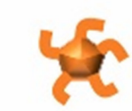

assembly reaction II drug loading

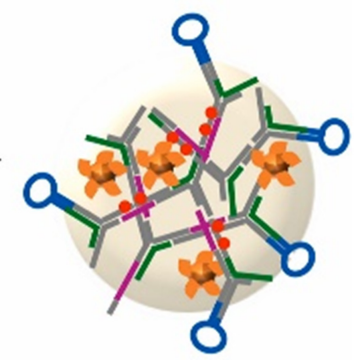

nanocomplex

B

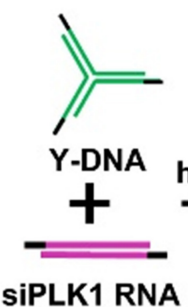

SiPLK1 RNA
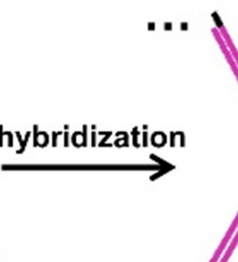

Öranched-DNARNA

$\mathrm{HO} \mathrm{OH} \mathrm{HOOHO}_{\mathrm{OH}}$

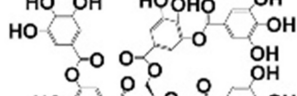

Ho $00^{\circ}$

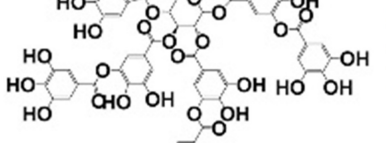

HO

$\mathrm{HO} \mathrm{OH}$

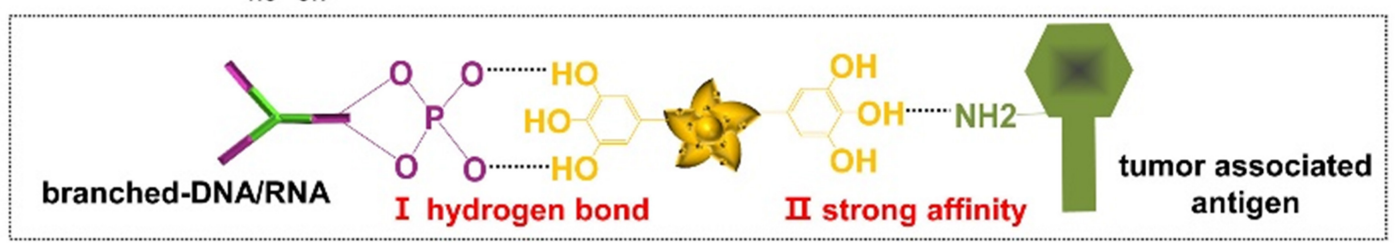

FIGURE 6 | Nanoassembly via TA mediated nucleic acid assembly. (A) DNA-polyphenol nanocomplex for efficient DNAzyme and antisense DNA delivery. Adapted with permission from ref. Han et al. (2021a). Copyright 2021, Elsevier Publishing Group. (B) Nucleic acid nanocomplex through TA mediating self-assembly for smart drug delivery and gene therapy. Adapted with permission from ref. Han et al. (2021b). Copyright 2021, Elsevier Publishing Group. 
DNAsomes through amphiphilicity-driven self-assembly (Thelu et al., 2017). These DNAsomes exhibited remarkable molecular recognition between $\beta$-CD-functionalized DNA (acting as the host molecule) and Ad-grafted hydrophobic segment (acting as the guest molecule). Subsequently, they further constructed nanogel with excellent cell permeability via self-assembly of $\beta$-CD-modified X-DNA or Y-DNA and Ad-grafted eight-arm star polyethylene glyco (PEG) polymer, showing its great potential in nucleic acid drugs delivery. Ding group have made great progress in the field of multifunctional nucleic acid nanostructures to deliver gene therapy drugs.

In 2019, Liu et al. (2019) further utilized tile-mediated assembly of $\beta$-CD-Ad and branched DNA to construct DNA-supermolecule hybrid nanostructure, achieving sgRNA/Clustered regularly interspaced short palindromic repeats associated 9 (Cas9)/antisense DNA delivery (Figure 5A). They used the circular supramolecular $\beta-C D$ as the core to covalently cross link nucleic acids by virtue of copper-free click chemistry to prepare branched DNA with seven-arm structures. Meanwhile, the $3^{\prime}$-end of the sgRNA (targeting the tumor-related gene PLK1) was extended to construct an RNA strand $\left(\operatorname{sgRNA} \mathrm{N}_{L}\right)$, which is complementary to ASO. Antisense (AS, targeting the tumor-related gene PLK1) modified by disulfide bonds at both ends was introduced as a linker for coassembly of the gene editing component (sgRNA/Cas9) and the gene silencing component (AS). An Ad-conjugated aptamer and Ad-modified hyaluronic acid (HA) endowed the nanoplatform with tumor-targeting and endosomal escape capabilities through host-guest interaction, respectively. Subsequently, sgRNA/Cas9/AS dissociated into sgRNA/Cas9 complex and antisense in response to intracellular GSH and RNase $H$, exhibiting excellent PLK1 gene silencing and potent antitumor effect. Also, based on this strategy, a coassembled nanoplatform of branched antisense DNA and siRNA was constructed for combined gene silencing in vitro and in vivo (Figure 5B; Liu et al., 2021). Covalently binding branched antisense can effectively capture the $3^{\prime}$-end siRNA to form NPs with controllable size. Second, biocompatible nucleic acid nanostructures can be functionalized with targeting groups and endoplasmic escape components through host-guest interactions. Finally, multifunctional nucleic acid nanocarriers can be digested by endogenous $\mathrm{RNase} H$ to effectively release nucleic acid products (branched antisense and siRNA). This is the biocompatible multifunctional nucleic acid nanoplatform. This novel strategy used skillfully nucleic acid self-assembly and host-guest interaction to combine DNA with peptide, realizing the multi-functionalization of the nucleic acid delivery vector and providing a new frontier of nucleic acid delivery.

Natural polyphenol originated from bioactive components of plant-based food. The typical biological role of polyphenol is reflected in the therapeutical functions for cancer therapy, such as anti-inflammation, anti-oxidation, and anti-apoptosis. In addition, the structural features of polyphenol also endowed strong interactions with nucleic acids to fabricate DNA-organic formulations, which demonstrated sufficient loading efficiency of gene drugs. In this regard, we employed tannic acid (TA) to mediate the assembly process of the dynamic nanocomplex system (Figure 6A; Han et al., 2021a). In the composite system, Y-shaped DNA and linear DNA assembled into branched skeleton through complementary base pairing and formed nanocomposites with TA driven by hydrogen bonding. The acidic environments in the lysosome would induce the degradation of TA and resulted in the disassembly of the nanocomplex to release branched DNA to the cytoplasm. In response to intracellular GSH and DNase I, branched DNA was cleaved to antisense DNA and DNAzyme segments, which afterward suppressed cell proliferation and cell migration, respectively. Notably, TAs also facilitated cell apoptosis to enhance the therapeutic effects. DNApolyphenol coordinated nanocomplex ensured extremely high loading efficiency and achieved the responsive and sequential release of gene/drugs. The incorporation of TAs into the structural composition offered adequate controllability and flexibility in the design of drug delivery carriers, which showed excellent efficacy at in vivo levels.

Despite the diverse drug nanocarriers, the limited targeting specificity and blood stability generally resulted in poor enrichments to tumor sites. Inspired by the camouflage mechanism of cancer cells against the immune system, the cell membrane coating strategy provided feasible means to manipulate the fates of original nanocarriers. Due to the structural affinity between TAs and proteins, we further coated DNA-polyphenol nanocomplex with the cell membrane (Figure 6B; Han et al., 2021b). The homotypic targeting capability derived from the membrane improved the blood circulation stability of nanocomplex and reduced the clearance by the reticular endothelial system. In the cancer cells, the acid-responsive degradation served as the trigger for the decomposition of the nanocomplex, which further led to the controllable release of the functional nucleic acid moiety. In this dynamic complex system, TAs connected the outer shell of the cell membrane and inner nucleic acids components, which realized efficient drug delivery while demonstrated specific and on-demand release behaviors. TAs as the natural polyphenol are arguably promising candidates for engineering intelligent drug delivery platform in the field of precision medicine.

\section{CONCLUSION AND OUTLOOK}

In this study, we have summarized the recent progress in DNA functional nanostructures for the delivery of nucleic acid-based drugs. Due to the sequence programmability of DNA, DNAbased nanostructures are highly designable at the molecular level, which facilitates to realize controllable assembly and disassembly of nucleic acid-based drugs in specific application scenarios. Furthermore, DNA molecules are easily combined with other assembly systems to achieve functional scalability and thus to improve delivery performances. Despite the rapid development in DNA-based gene delivery nanomaterials, the clinical transformation of those approaches still confronted with several challenges, including standardized manufacture, stability, biosafety concerns, and socioeconomic issues.

The standardized manufacture of DNA-based gene delivery nanomaterials needs further exploration. Although DNA has controlled structural conformation and sequence programmability, the synthetic efficiency of DNA assembly 
is still relatively low due to steric hindrance and limited atom utilization, which impedes standardized mass production. Optimizing and controlling the assembly condition might be an avenue to improve the efficiency of constituent atom utilization.

To enhance the delivery and therapeutic efficacy, DNAbased nanomaterials need prolonged blood circulation time to achieve efficient accumulation at lesion sites. However, the prolonged circulation time may cause degradation concerns in vivo. Therefore, the stability of DNA nanomaterials is vital for the effective delivery of nucleic acid-based drugs. Chemical modifications of DNA and integration with other materials have been proposed to be effective to enhance resistance to enzymatic degradation and improve the stability of DNAbased nanomaterials.

DNA is an endogenous substance with excellent biosafety and biocontrollable degradability. Studies have shown good biosafety of DNA-based nanomaterials, whereas limited investigation has involved long-term biosafety issues. Moreover, immunostimulatory sequence-integrated DNA nanomaterials may induce immune responses. Rational DNA sequence design of DNA-based nanomaterials is needed to guarantee the long-term biological security of DNA nanomaterials. However, there might be immune responses toward DNA, which is hard to completely avoid on occasions.

DNA used for the fabrication of DNA nanomaterials is mainly chemically synthesized currently. However, the length of DNA is limited to below 200 nucleotides. Therefore, the high cost of DNA-based nanomaterials remains a major challenge, which could cause socioeconomic issues to satisfy large-scale production for nucleic acid delivery. Exploring more effective methods to synthesize DNA and developing cost-effective storage

\section{REFERENCES}

Amo-Ochoa, P., and Zamora, F. (2014). Coordination polymers with nucleobases: from structural aspects to potential applications. Coord. Chem. Rev. 276, 34-58. doi: 10.1016/j.ccr.2014.05.017

Ashton, P. R., Königer, R., Stoddart, F., Alker, D., and Harding, V. D. (1996). Amino acid derivatives of $\beta$-cyclodextrin. J. Org. Chem. 61, 903-908. doi: 10 . 1021/jo951396d

Chen, G., Liu, D., He, C., Gannett, T. R., Lin, W., and Weizmann, Y. (2015). Enzymatic synthesis of periodic DNA nanoribbons for intracellular $\mathrm{pH}$ sensing and gene silencing. J. Am. Chem. Soc. 137, 3844-3851. doi: 10.1021/ja512665z

Chin, S. M., Synatschke, C. V., Liu, S., Nap, R. J., Sather, N. A., Wang, Q., et al. (2018). Covalent-supramolecular hybrid polymers as muscle-inspired anisotropic actuators. Nat. Commun. 9:2395. doi: 10.1038/s41467-018-04800-w

Davis, M. E., and Brewster, M. E. (2004). Cyclodextrin-based pharmaceutics: past, present and future. Nat. Rev. Drug Discov. 3, 1023-1035. doi: 10.1038/nrd1576

Ding, F., Mou, Q., Ma, Y., Pan, G., Guo, Y., Tong, G., et al. (2018). A crosslinked nucleic acid nanogel for effective siRNA delivery and antitumor therapy. Angew. Chem. Int. Ed. Engl. 57, 3064-3068. doi: 10.1002/anie.201711242

Dong, Y., Yao, C., Zhu, Y., Yang, L., Luo, D., and Yang, D. (2020). DNA functional materials assembled from branched DNA: design, synthesis, and applications. Chem. Rev. 120, 9420-9481. doi: 10.1021/acs.chemrev.0c00294

Han, J., Cui, Y., Gu, Z., and Yang, D. (2021a). Controllable assembly/disassembly of polyphenol-DNA nanocomplex for cascade-responsive drug release in cancer cells. Biomaterials 273:120846. doi: 10.1016/j.biomaterials.2021.120846

Han, J., Cui, Y., Li, F., Gu, Z., and Yang, D. (2021b). Responsive disassembly of nucleic acid nanocomplex in cells for precision medicine. Nano Today 39:101160. doi: 10.1016/j.nantod.2021.101160 approaches are highly necessary. The techniques to obtain biomass DNA may address this cost concern. For example, the plasmid containing target DNA fragments is transfected into bacteria, such as Escherichia coli (E. coli) to amplify DNA along with the growth of the bacteria. Another available technique is using PCR to amplify specific DNA fragments, which would reduce the cost of DNA production.

In summary, it is firmly believed that nucleic acid drug therapeutics are doomed to generate a revolutionary impact on disease treatment in the near future.

\section{AUTHOR CONTRIBUTIONS}

ZL, YZ, and FL: writing - original draft preparation. ZL and FL: writing - review and editing. FL: supervision. All authors contributed to the article and approved the submitted version.

\section{FUNDING}

This work was supported in part by the National Natural Science Foundation of China (Grant Nos. 21905196 and 31971305) and Tianjin Natural Science Foundation (Basic Research Plan, Grant No. 19JCQNJC01900).

\section{ACKNOWLEDGMENTS}

We thank Yuhang Dong and Rui Zhang at the Tianjin University for their help on the discussion.

Hendrikse, S. I. S., Gras, S. L., and Ellis, A. V. (2019). Opportunities and challenges in DNA-hybrid nanomaterials. ACS NANO 13, 8512-8516. doi: 10. 1021/acsnano.9b06186

Hu, C., Wu, J., Wei, T., Zhan, W., Qu, Y., Pan, Y., et al. (2018). A supramolecular approach for versatile biofunctionalization of magnetic nanoparticles. J. Mat. Chem. B 6, 2198-2203. doi: 10.1039/c8tb00490k

Huo, S., Jin, S., Ma, X., Xue, X., Yang, K., Kumar, A., et al. (2014). Ultrasmall gold nanoparticles as carriers for nucleus-based gene therapy due to size-dependent nuclear entry. ACS NANO 8, 5852-5862. doi: 10.1021/nn5008572

Huo, S. D., Gong, N. Q., Jiang, Y., Chen, F., Guo, H. B., Gan, Y. L., et al. (2019). Gold-DNA nanosunflowers for efficient gene silencing with controllable transformation. Sci. Adv. 5:eaaw6264. doi: 10.1126/sciadv.aaw6264

Jiang, Z., and Thayumanavan, S. (2020). Noncationic material design for nucleic acid delivery. Adv. Therap. 3:1900206. doi: 10.1002/adtp.201900206

Lee, H., Lytton-Jean, A. K., Chen, Y., Love, K. T., Park, A. I., Karagiannis, E. D., et al. (2012). Molecularly self-assembled nucleic acid nanoparticles for targeted in vivo siRNA delivery. Nat. Nanotechnol. 7, 389-393. doi: 10.1038/nnano.2012.73

Leininger, S., Olenyuk, B., and Stang, P. J. (2000). Self-assembly of discrete cyclic nanostructures mediated by transition metals. Chem. Rev. 100, 853-908.

Li, F., Tang, J., Geng, J., Luo, D., and Yang, D. (2019). Polymeric DNA hydrogel: design, synthesis and applications. Prog. Polym. Sci. 98:101163. doi: 10.1016/j. progpolymsci.2019.101163

Li, M., Wang, C., Di, Z., Li, H., Zhang, J., Xue, W., et al. (2019). Engineering multifunctional DNA hybrid nanospheres through coordination-driven selfassembly. Angew. Chem. Int. Ed. 58, 1350-1354. doi: 10.1002/anie.201810735

Li, F., Yu, W., Zhang, J., Dong, Y., Ding, X., Ruan, X., et al. (2021). Spatiotemporally programmable cascade hybridization of hairpin DNA in 
polymeric nanoframework for precise siRNA delivery. Nat. Commun. 12:1138. doi: 10.1038/s41467-021-21442-7

Li, J., Zheng, C., Cansiz, S., Wu, C., Xu, J., Cui, C., et al. (2015). Self-assembly of DNA nanohydrogels with controllable size and stimuli-responsive property for targeted gene regulation therapy. J. Am. Chem. Soc. 137, 1412-1415. doi: $10.1021 /$ ja512293f

Liu, J., Lu, X., Wu, T., Wu, X., Han, L., and Ding, B. (2021). Branched antisense and siRNA co-assembled nanoplatform for combined gene silencing and tumor therapy. Angew. Chem. Int. Ed. 60, 1853-1860. doi: 10.1002/anie.202011174

Liu, J., Song, L., Liu, S., Zhao, S., Jiang, Q., and Ding, B. (2018). A tailored DNA nanoplatform for synergistic RNAi-/chemotherapy of multidrug-resistant tumors. Angew. Chem. Int. Ed. Engl. 57, 15486-15490. doi: 10.1002/anie. 201809452

Liu, J., Wu, T., Lu, X., Wu, X., Liu, S., Zhao, S., et al. (2019). A self-assembled platform based on branched DNA for sgRNA/Cas9/antisense delivery. J. Am. Chem. Soc. 141, 19032-19037. doi: 10.1021/jacs.9b09043

Luckerath, T., Koynov, K., Loescher, S., Whitfield, C. J., Nuhn, L., Walther, A., et al. (2020). DNA-polymer nanostructures by RAFT polymerization and polymerization-induced self-Assembly. Angew. Chem. Int. Ed. 59, 1547415479. doi: 10.1002/anie.201916177

Lv, J., Dong, Y., Gu, Z., and Yang, D. (2020). Programmable DNA nanoflowers for biosensing, bioimaging, and therapeutics. Chem. Eur. J. 26, 14512-14524. doi: $10.1002 /$ chem. 202002242

Pan, M., Jiang, Q., Sun, J., Xu, Z., Zhou, Y., Zhang, L., et al. (2020). Programming DNA nanoassembly for enhanced photodynamic therapy. Angew. Chem. Int. Ed. 59, 1897-1905. doi: 10.1002/anie.201912574

Pan, X., Lathwall, S., Mack, S., Yan, J., Das, S. R., and Matyjaszewski, K. (2017). Automated synthesis of well-defined polymers and biohybrids by atom transfer radical polymerization using a DNA synthesizer. Angew. Chem. Int. Ed. 56, 2740-2743. doi: 10.1002/anie.201611567

Pelegri-O'Day, E. M., and Maynard, H. D. (2016). Controlled radical polymerization as an enabling approach for the next generation of protein-polymer conjugates. Accounts Chem. Res. 49, 1777-1785. doi: $10.1021 /$ acs.accounts.6b00258

Singh, Y., Murat, P., and Defrancq, E. (2010). Recent developments in oligonucleotide conjugation. Chem. Soc. Rev. 39, 2054-2070. doi: 10.1039/ b911431a
Thelu, H. V. P., Albert, S. K., Golla, M., Krishnan, N., Ram, D., Srinivasula, S. M., et al. (2017). Size controllable DNA nanogels from the self-assembly of DNA nanostructures through multivalent host-guest interactions. Nanoscale 10, 222-230. doi: 10.1039/c7nr06985e

Vaughan, H. J., Green, J. J., and Tzeng, S. Y. (2020). Cancer-targeting nanoparticles for combinatorial nucleic acid delivery. Adv. Mater. 32:e1901081. doi: 10.1002/ adma.201901081

Wang, J., Yu, S., Wu, Q., Gong, X., He, S., Shang, J., et al. (2021). A self-catabolic multifunctional DNAzyme nanosponge for programmable drug delivery and efficient gene silencing. Angew. Chem. Int. Ed. 60, 10766-10774. doi: 10.1002/ anie. 202101474

Wu, X., Wu, T., Lu, J., and Ding, B. (2020). Gene therapy based on nucleic acid nanostructure. Adv. Healthc. Mater. 9:e2001046. doi: 10.1002/adhm.202001046

Yuan, Y., Gu, Z., Yao, C., Luo, D., and Yang, D. (2019). Nucleic acid-based functional nanomaterials as advanced cancer therapeutics. Small 15:e1900172. doi: $10.1002 /$ smll.201900172

Zhang, J., Li, F., and Yang, D. (2019). DNA: from carrier of genetic information to polymeric materials. Trans. Tianjin Univ. 25, 301-311. doi: 10.1007/s12209019-00188-w

Conflict of Interest: The authors declare that the research was conducted in the absence of any commercial or financial relationships that could be construed as a potential conflict of interest.

Publisher's Note: All claims expressed in this article are solely those of the authors and do not necessarily represent those of their affiliated organizations, or those of the publisher, the editors and the reviewers. Any product that may be evaluated in this article, or claim that may be made by its manufacturer, is not guaranteed or endorsed by the publisher.

Copyright (c) $2021 \mathrm{Lv}, \mathrm{Zhu}$ and Li. This is an open-access article distributed under the terms of the Creative Commons Attribution License (CC BY). The use, distribution or reproduction in other forums is permitted, provided the original author(s) and the copyright owner(s) are credited and that the original publication in this journal is cited, in accordance with accepted academic practice. No use, distribution or reproduction is permitted which does not comply with these terms. 